\title{
Antibody response to BTN162b2 mRNA vaccination in naiive versus SARS-CoV-2 infected subjects with and without waning immunity
}

\section{Luca Dalle Carbonare}

University of Verona https://orcid.org/0000-0003-3263-6671

Maria Teresa Valenti

Department of Medicine, University of Verona; Policlinico GB Rossi-AOUI Verona

\section{Zeno Bisoffi}

IRCCS Sacro Cuore Don Calabria Hospital

\section{Chiara Piubelli}

IRCCS Sacro Cuore Don Calabria Hospital https://orcid.org/0000-0002-5739-3795

\section{Massimo Pizzato}

University of Trento https://orcid.org/0000-0002-9491-1483

Silvia Accordini

University of Trento

\section{Sara Mariotto}

University of Verona

\section{Sergio Ferrari}

University of Verona

Arianna Minoia

University of Verona Jessica Bertacco

University of Verona

Veronica Li Vigni

University of Verona

Gianluigi Dorelli

University of Verona

Ernesto Crisafulli

University of Verona

\section{Daniela Alberti}

University of Verona

\section{Laura Masin}

University of Verona

\section{Silvia Longoni}


IRCCS Sacro Cuore Don Calabria Hospital

\section{Lucia Lopalco}

San Raffaele Scientific Institute

Alberto Beretta

Covi2 Technologies Srl

Natalia Tiberti

IRCCS Sacro Cuore Don Calabria Hospital

Donato Zipeto ( $\nabla$ donato.zipeto@univr.it)

University of Verona https://orcid.org/0000-0002-2168-4144

\section{Article}

Keywords: SARS-CoV-2, antibodies, mRNA vaccine

Posted Date: April 30th, 2021

DOl: https://doi.org/10.21203/rs.3.rs-440410/v1

License: (c) (1) This work is licensed under a Creative Commons Attribution 4.0 International License. Read Full License

Version of Record: A version of this preprint was published at Communications Medicine on October 13th, 2021. See the published version at https://doi.org/10.1038/s43856-021-00039-7. 
1 Antibody response to BTN162b2 mRNA vaccination in naïve versus SARS-CoV-

$2 \quad 2$ infected subjects with and without waning immunity

3

4 Authors

5 Luca Dalle Carbonare ${ }^{1}$, Maria Teresa Valenti ${ }^{1}$, Zeno Bisoffi ${ }^{2,3}$, Chiara Piubelli ${ }^{2}$,

6 Massimo Pizzato ${ }^{4}$, Silvia Accordini ${ }^{4}$, Sara Mariotto ${ }^{5}$, Sergio Ferrari ${ }^{5}$, Arianna

7 Minoia $^{1}$, Jessica Bertacco ${ }^{1}$, Veronica Li Vigni ${ }^{1}$, Gianluigi Dorelli ${ }^{1}$, Ernesto

8 Crisafulli $^{1}$, Daniela Alberti ${ }^{5}$, Laura Masin ${ }^{5}$, Natalia Tiberti $^{2}$, Silvia Stefania Longoni ${ }^{2}$,

9 Lucia Lopalco ${ }^{6}$, Alberto Beretta ${ }^{7}$, Donato Zipeto ${ }^{5 *}$

10

$11 \quad{ }^{1}$ Department of Medicine, University of Verona

$12{ }^{2}$ Department of Infectious, Tropical Diseases and Microbiology, IRCCS Sacro Cuore

13 Don Calabria Hospital, Negrar (Verona)

$14{ }^{3}$ Department of Diagnostics and Public Health, University of Verona

$15{ }^{4}$ Department of Cellular, Computational and Integrative Biology, University of

16 Trento, Povo (Trento)

$17{ }^{5}$ Department of Neuroscience, Biomedicine and Movement Sciences, University of

18 Verona

$19{ }^{6}$ Division of Immunology, Transplantation and Infectious Diseases, San Raffaele

20 Scientific Institute, Milan

$21 \quad{ }^{7}$ Covi2 Technologies Srl, Novara

22 All in Italy

23

$24 *$ Corresponding author 
Abstract

We profiled antibody responses in a cohort of recipients of the BTN162b2 mRNA vaccine who were either immunologically naïve $(n=50)$ or had been previously infected with SARS-CoV-2 (n=51). Of the previously infected, 25 and 26 were infected during the first and second pandemic waves in Italy, respectively; the majority of those from the first wave had corresponding waning immunity with low to undetectable levels of anti-S antibodies and low anti-N antibodies. We observed in recipients who had been previously infected that spike-specific IgG and pseudovirus neutralization titers were rapidly recalled by a single vaccine dose to higher levels than those in naïve recipients after the second vaccine dose, irrespective of waning immunity. In all recipients, a single vaccine dose was sufficient to induce a potent IgA response that was not associated with serum neutralization titers.

As we write, four COVID-19 vaccines have been authorized for use by FDA and/or EMA, and additional vaccine candidates are under evaluation. The authorized vaccines are based on the use of $\mathrm{mRNA}^{1}$ or adenoviral vectors ${ }^{2}$ that induce the expression of the SARS-CoV-2 spike protein. Apart from the Johnson \& Johnson adenovirus-based vaccine that requires only a single dose, all other vaccines are based on a double dose regimen to maximize their efficacy.

Increasingly available anti-SARS-CoV-2 antibody and virus-specific T cell data support a strategy that previously infected (P.I.) vaccine recipients have sufficient immune response from only one vaccine dose ${ }^{3-6}$ this would have significant impact on global vaccine supply. Spike-specific IgG antibody levels and ACE2 antibody binding inhibition responses elicited by a single vaccine dose in individuals with prior SARS-CoV-2 infection (P.I.) were similar to those seen after two doses of vaccine in 
50 individuals without prior infection (naïve) ${ }^{7}$. In another study, the antibody titers of

51 recipients with preexisting immunity were 10 to 45 -fold higher than naive recipients

52 at the same time points after the first vaccine dose of vaccine, and no increases in

53 antibody titers were observed in P.I. recipients who received the second vaccine dose

$54 \quad$. Similarly, Bradley et al reported that after the first vaccine dose, recently infected

55 P.I. recipients had higher titers of antibodies to the S1 and S2 subunit and the

56 receptor-binding domain (RBD) of the spike protein compared to naïve recipients

57 who had received the two doses of vaccine ${ }^{9}$. Interestingly, at baseline, naïve

58 recipients exhibited a significant level of reactivity to the S2 subunit, suggesting a

59 pre-existing cross-reactive response to common coronavirus infections ${ }^{9}$. In another

60 study, antibody binding to a trimeric spike protein and live-virus neutralization assays

61 performed on a cohort of volunteers who received one dose of an mRNA vaccine

62 showed a rapid response in P.I. recipients with antibody titers raising at 7 days and

63 reaching a peak 10-14 days post-vaccination, a kinetic that was significantly faster

64 than that observed in naïve individuals ${ }^{10}$. In addition, two of these studies reported

65 that vaccine reactogenicity was more prominent in P.I. individuals after the first dose

66 but similar between the two groups after the second dose ${ }^{7,8}$. A cautionary tale for the

67 use of a two doses regimen in P.I. individuals was raised by Levi et al who considered

68 the possibility of antibody-dependent enhancement ${ }^{11}$ or antigen exhaustion as a result

69 of an over-boosting of immune responses ${ }^{12}$. The lack of an established correlate of

70 protection against disease and/or infection adds further complexity. The emergence of

71 SARS-CoV-2 variants is also a concern, and Stamatatos et al. highlighted the

72 importance of two dose regimens in both naïve and P.I. individuals to achieve cross-

73 variant neutralizing antibodies ${ }^{13}$. An additional component of the immune response to

74 SARS-CoV-2 that could influence the outcome of vaccination is the presence in most 
75 SARS-CoV-2 naïve individuals of variable levels of pre-existing immunity to spike

76 protein epitopes that are shared with other common human coronaviruses (hCoVs);

$77 \quad 14,15$ these have been suggested to be potentially protective or pathogenic and may

78 shape the kinetic and potency of the immune response to the vaccine ${ }^{16-19}$.

79 Here we report data from a serological profile of a cohort of 101 naïve and P.I.

80 recipients who received both doses of Pfizer-BioNtech BNT162b2 mRNA vaccine.

81 We took advantage of the availability of two different sub-groups of P.I. recipients

82 who experienced a SARS-CoV-2 infection during the first wave (Spring 2020) and

83 the second wave (Autumn 2020) of the pandemic in Northern Italy to investigate the

84 different effects of vaccination in recipients with recent-active or past-waning

85 immunity. Antibody levels were measured at three time points: prior to first

86 vaccination (T0), prior to second vaccination (T1), and three weeks after the second

87 vaccination (T2). As the BNT162b2 vaccine is expected to elicit only IgG-S

88 antibodies, we also tested all subjects for the presence of IgG-nucleocapsid (IgG-N)

89 antibodies to identify recipients with past infection. The IgG-N antibody test is also a

90 reliable marker of enduring immunity to SARS-CoV-2 ${ }^{20}$ and as such can be used to

91 monitor waning immunity. Since natural infection with SARS-CoV-2 is often

92 followed by a rapid rise in IgG antibodies that can occur concomitantly or even before

93 the appearance of IgM antibodies ${ }^{14,21-23}$ we tested whether a similar pattern would

94 follow vaccination by measuring both $\mathrm{IgG}$ antibodies specific for the RBD of the

95 spike protein (IgG-S(RBD)) and IgM spike-specific antibodies (IgM-S).

96 IgA have also been implicated in protective immunity to SARS-CoV-2, but, to the

97 best of our knowledge, there are no available data on the IgA response following

98 vaccination and no potential association between vaccine-induced IgA response and

99 serum virus-neutralizing activity. During natural SARS-CoV-2 infection, IgA 
100 responses precede $\operatorname{IgG}$ responses ${ }^{24-26}$. Whether this is also the case after vaccination

101 is unknown. We therefore tested all sera for the presence of IgG-N, IgM-S, IgG-

$102 \mathrm{~S}(\mathrm{RBD})$, and IgA-S as well as for the presence of virus-neutralizing activity as

103 measured in a pseudovirus neutralization assay.

104 We enrolled 101 healthcare workers with (P.I.) or without (naïve) preexisting

105 immunity to SARS-CoV-2. Of the 51 P.I. vaccinees, 25 had been infected during the

106 first wave and 26 during the second wave. All subjects received the first vaccine dose

107 (BNT162b2 mRNA, Pfizer-BioNTech) in January 2021. The two groups were

108 homogeneous in age and sex (Table 1).

109 IgG-N antibody testing was negative in all naïve recipients and positive in $24 / 51$

110 (47\%) P.I. recipients (Fig. 1A). A single subject, originally classified as naïve, who

111 resulted negative at baseline but highly positive at T1 and T2 for the presence of IgG-

$112 \mathrm{~N}$ was excluded from the analysis. In the P.I group, at baseline, 7/25 (28\%) and 17/26

$113(65 \%)$ of those who were infected during the first and second waves, respectively,

114 were positive for IgG-N antibodies, consistent with a trend toward waning immunity

115 in recipients infected during the first wave (Fig. 2A and Table 2).

116 IgM-S antibodies measured before vaccination (T0) showed a similar pattern with

$1178 / 25(32 \%)$ and $16 / 26(61 \%)$ testing positive at baseline in the first and second wave

118 P.I. recipients, respectively (Fig. 2B and Table 2). Following vaccination, 27/50

119 (54\%) naïve recipients became IgM-S positive after the first dose, and 29/49 (59\%, 20

120 of whom were already positive after the first dose) were positive after the second dose

121 with no significant increase in antibody titers compared to baseline (Fig. 1B and Table

122 2). In P.I. recipients from the first or second wave, there was no significant difference

123 in the IgM-S response after vaccination (Fig. 2B and Table 2). 
124 IgG-S(RBD) were detectable after the first vaccine dose in 49/50 (98\%) naïve

125 recipients but with very low titers that were boosted by the second vaccine dose

126 resulting in a highly significant increase $(\mathrm{p}<0.0001)$ (Fig. 1D and Table 2). Forty-

127 six/51 (90\%) P.I. recipients showed low IgG-S(RBD) titers at baseline (Fig. 1D and

128 Table 2), and the first vaccine dose induced a strong increase in IgG-S(RBD) reaching

129 levels that were 12-fold higher than titers observed in naïve subjects after the first

130 dose (naïve T1: 1673 \pm 1787 ; P.I. T1: 20131 $\pm 18990 ; \mathrm{p}<0.0001$ ) and 1.9-fold higher

131 when comparing P.I. and naïve recipients after the second dose (naïve T2:

$13219551 \pm 10941$; P.I. T2: $37607 \pm 22895 ; \mathrm{p}=0.4583$, ns), whereas it was similar when

133 comparing P.I. recipients after the first dose to naïve recipients after the second dose

134 (naïve T2 19551 \pm 10941 ; P.I. T1: 20131 $\pm 18990, \mathrm{p}>0.9999$, ns). The second vaccine

135 dose in P.I. recipients did not result in a significant increase in antibody titers

136 consistent with data reported by other authors ${ }^{7-9,27}$. Comparison of P.I. recipients

137 infected during the two waves did not show any significant difference in the responses

138 to the first and second vaccine dose (Fig. 2D and Table 2), although there was an

139 unexpectedly higher (although not statistically significant) response to the first dose in

140 those infected during the first wave compared with those infected during the second

141 wave, with recipients showing binding titers as high as $80000 \mathrm{AU} / \mathrm{ml}$.

142 The pseudovirus neutralization assay (TCID50) essentially mirrored the results of the

143 IgG-S(RBD) assay with 47/50 (94\%) naïve recipients showing a weak but positive

144 score (>120 for TCID50) after the first dose and 49/49 (100\%) after the second dose,

145 but with a highly significant $(\mathrm{p}<0.0001)$ increase in neutralizing titers (Fig. 1E). In

146 P.I. recipients, we observed the same pattern seen in the IgG-S(RBD) assay with an

147 efficient boost of neutralizing antibodies after the first dose $(p<0.0001)$ and no further

148 increase after the second dose (Fig. 1E). 
149 A correlation analysis between the IgG-S(RBD) and the pseudovirus neutralization assay (TCID50) confirmed a strong association between serum IgG-S(RBD) and

151 neutralizing titers (Fig. 3A) consistent with other reports showing a major role played

152 by RBD-specific antibodies in virus neutralization ${ }^{28}$. Results of the two waves P.I.

153 cohorts also mirrored those obtained with the IgG-S(RBD) and evidenced a

154 surprisingly, although not statistically significant, higher virus-neutralization response

155 in P.I. recipients of the first wave compared with the second, with TCID50 titers as

156 high as $1 / 4000$ (Fig. 2E). These data are strongly suggestive of the persistence of

157 memory B cell responses that can be rapidly recalled by a single vaccine dose after 9-

15810 months from primary infection even in the absence of detectable serum IgG-

$159 \mathrm{~S}(\mathrm{RBD})$ antibodies. It is also conceivable that natural infection with SARS-CoV-2

160 may prime the immune system to produce antibody specificities other than RBD that

161 can be readily recalled by a single dose of vaccine.

162 The potential implication of cross-reactive immunity to other coronaviruses in the

163 response to vaccination is supported by an unexpected feature that emerged from our

164 data: the unconventional isotype pattern observed in both naïve and P.I recipients. In

165 the naïve recipients, after the first dose, when the canonical primary immune response

166 is expected to generate IgM first followed by IgG, only $27 / 50(54 \%)$ recipients were

167 positive for IgM-S with no further increase after the second dose $(29 / 49 ; 59 \%)$,

168 whereas 49/50 (98\%) and 49/49 (100\%) naïve recipients scored positive for IgG-

$169 \mathrm{~S}(\mathrm{RBD})$ after the first and second dose respectively (Table 2). Twenty-three/50 (46\%)

170 naïve recipients showed an IgG-S(RBD) positive test but were negative for IgM-S,

$17127 / 50(54 \%)$ were positive for both IgG-S(RBD) and IgM-S, and none were positive

172 for IgM-S and negative for IgG-S(RBD) (Table 3). This isotype pattern is consistent 
173 with that of an anamnestic response sustained by memory B cells specific for spike 174 epitopes shared with other common human coronaviruses ${ }^{14,15}$.

175 At baseline, IgA-S were detected in none of the naïve recipients, in 18/25 (72\%) of 176 the P.I. recipients who were infected during the first wave, and in 22/26 (84\%) of 177 those infected during the second wave (Fig. 1C and Table 2). The first vaccine dose 178 resulted in a significant increase $(\mathrm{p}<0.0001)$ in IgA-S titers in both naïve and P.I. 179 recipients (Fig. 1C). The second dose further boosted the IgA-S titers in naïve 180 recipients $(\mathrm{p}<0.0001)$ but led to a significant reduction in P.I. recipients $(\mathrm{p}=0.0099)$.

181 The decline in IgA titers after the second vaccination in P.I. recipients was not related 182 to the time from infection, as it was observed in both subjects infected during the first 183 and second wave and most likely represents a response to the vaccination (Fig. 2C). A 184 correlation analysis between the IgA-S and IgG-S(RBD) titers at baseline and $\mathrm{T} 1$ and 185 T2 revealed in naïve subjects the appearance of high IgA titers after the first vaccine 186 dose followed by a significant increase in IgG-S(RBD) titers only after the second 187 dose (Fig. 3B). In P.I. recipients, the first and second dose boosted both IgA-S and 188 IgG-S(RBD) titers (Fig. 3B). We did not observe a correlation between IgA-S titers 189 and virus-neutralization titers (TCID50) both in naïve and P.I. recipients (Fig. 3C). On 190 the other hand, the early increase in IgA after the first dose that preceded the increase 191 in IgG-S(RBD) titers after the second vaccine dose is consistent with what has been 192 observed during natural SARS-CoV-2 infection where IgAs precede IgGs ${ }^{25,26,29}$. The 193 presence of IgA-S in a significant proportion of P.I. recipients at baseline is also 194 consistent with the slower waning of IgA compared to IgG observed in convalescent 195 patients ${ }^{30}$.

196 We next examined the influence of gender in the IgG-S(RBD) and IgA-S responses to 197 vaccination. In the naïve recipients, there were no differences in the kinetic and size 
of IgG-S(RBD) responses between males and females. In contrast, in the P.I group, males responded to the vaccine by producing higher titers of IgG-S(RBD) than females $(\mathrm{p}<0.05)$ (Fig. 4A). This difference was reflected by the neutralization assay,

201 which showed higher neutralizing titers in males than in females (Fig.4B). The

202 differences between the two groups were significant in male and female naïve IgA-S 203 responses at $\mathrm{T} 1(\mathrm{p}<0.05)$ and $\mathrm{T} 2(\mathrm{p}<0.01)$ (Fig.4C).

204 Taken together these data show that: 1) immunologically naïve recipients react to the 205 first dose of vaccine with a low-titer IgG-S(RBD) response that is then boosted by the 206 second dose; 2) in previously infected recipients one dose of vaccine is sufficient to 207 induce antibody titers that are higher than those observed in naïve recipients after the 208 second vaccine dose; 3 ) there is a good correlation between IgG-S(RBD) titers and 209 virus neutralizing titers, confirming that the IgG-S(RBD) testing is a proxy for virus

210 neutralization; 4) recipients who were infected with SARS-CoV-2 during the first

211 pandemic wave exhibit a rapid response to vaccination measured as both $\operatorname{IgG}-\mathrm{S}(\mathrm{RBD})$

212 binding titers and virus neutralizing titers; 5 ) in $46 \%$ of naïve recipients, IgG-S(RBD)

213 appear in the absence of IgM-S as if the response to vaccination was influenced by 214 previous antigen exposures; 6) in naïve recipients IgA-S appear before IgG-S(RBD)

215 after the first vaccine dose and are further boosted by the second dose; 7) in

216 previously infected recipients, IgA show a rapid, high titer response to the fist vaccine

217 dose that is followed by a decline with the second dose; 8) there is no correlation

218 between IgA-S antibody titers and virus neutralization; 9) neutralization titers and 219 IgG-S(RBD) were higher in previously infected male recipients compared to female 220 but not in naïve recipients.

221 Our findings expand on previous studies that indicated higher levels of anti-S 222 antibodies at baseline and after a single mRNA vaccine dose in previously infected 
223 individuals compared with those without prior infection, suggesting that a second

224 vaccine dose does not offer P.I. recipients a substantially greater benefit over a single

225 dose in antibody neutralization. The availability of two sub-groups of recipients who

226 had been infected during the first and second waves of the pandemic in Italy gave us

227 the additional opportunity to evaluate the effects of one dose versus two doses of

228 vaccination in the context of a past-waning immunity and compare it with that of

229 recent-active immunity. The lower IgG-N and IgG-S(RBD) antibody titers observed

230 at baseline in the first wave P.I. vaccines compared to the second wave P.I vaccines

231 (Fig. 2A) gave us confidence that the two cohorts were in two different stages of post-

232 infection immunity. However, when the IgA-S antibody titers were considered, no

233 substantial differences were observed between the two cohorts at baseline, indicating

234 that serum IgAs may be a better marker of long-lasting immunity and that

235 immunological memory may be more long-lasting than what previously estimated on

236 the basis of IgG antibody levels alone. This was further substantiated by the surprising

237 rapid recall of high $\mathrm{IgG}-\mathrm{S}(\mathrm{RBD})$ and virus neutralizing titers observed in first wave

238 P.I. recipients even in individuals with absent or very low serum IgG-S(RBD) levels.

239 Although not statistically significant, the response observed in the first wave P.I.

240 recipients was greater than that observed in the second wave P.I. recipients, probably

241 as a result of the persistence of memory B cell responses that can be rapidly recalled

242 by a single dose and consistent with recent data on the appearance in convalescent

243 patients of memory B cells with a turnover time of 6 months that express antibodies

244 with increased somatic hypermutations, neutralizing breadth, and potency ${ }^{31}$. The

245 possibility that natural infection with SARS-CoV-2 may prime the immune system to

246 produce antibody specificities other than RBD, further expanding the antibody

247 repertoire induced by vaccination, should also be considered. Our study did not 
address the epitope specificities of vaccination-induced antibodies, and additional studies addressing the fine specificities of vaccine-induced antibodies are warranted. A priming effect of previous exposures to common human coronaviruses on the immune response to the vaccine is suggested by our findings in the cohort of naïve recipients showing $\operatorname{IgG}-\mathrm{S}(\mathrm{RBD})$ antibodies in the absence of $\operatorname{IgM}-\mathrm{S}$ antibodies, an isotype pattern typical of anamnestic responses. Antibodies that cross-neutralize SARS-CoV-1, SARS-CoV-2, and other coronaviruses have been described that bind conserved epitopes of the hACE2 binding site showing extensive conservation among the SARS-like coronaviruses ${ }^{32}$. It remains to be determined whether the BTN162b2

257 mRNA vaccine is capable of eliciting these types of antibodies.

258 Our findings on the more rapid and potent IgA response compared with IgG responses 259 to the first vaccine dose parallel those in SARS-CoV-2 infected patients, where IgA 260 antibodies that bind to SARS-CoV-2 are produced rapidly after infection and remain 261 elevated in the plasma for at least 40 days after the onset of symptoms ${ }^{33}$. We did not 262 see a significant association between serum IgA and virus-neutralizing activity post263 vaccination, which, in contrast, has been reported in COVID-19 patients ${ }^{28}$. However, 264 it is plausible that the types of $\operatorname{IgA}$ antibodies elicited by intramuscular vaccination 265 may differ from the compartmentalized, mucosal immune response to natural 266 infection. Accordingly, SARS-CoV-2 specific plasma IgA monomers have been

267 shown to be two times less potent than IgG equivalents and, in contrast, IgA dimers, 268 the principal type of antibody in the nasopharynx, are 15 times more potent against 269 the same target as IgA monomers. ${ }^{33}$

270 Our data clearly show a rapid and potent induction of IgG-S(RBD) after a dose of 271 vaccine in individuals with past-waning immunity. Although a clear correlate of 272 protection from COVID-19 has not yet been identified, the recent findings by Zohar 
and colleagues that, notwithstanding equivalent $\operatorname{IgM}$ and $\operatorname{IgA}$ immunity to the virus

274 observed in different disease severity levels, rapid and potent IgG class switching is

275 associated with survival ${ }^{34}$ provide an additional argument in support of the use of a

276 single-dose vaccine regimen in individuals with prior SARS-CoV-2 infections.

277 Limitations of our study include the design (cross-sectional), the limited sample size,

278 the use of only one type of vaccine (Pfizer-BioNTech BNT162b2), the lack of

279 information on T-cell responses, and neutralization response against emerging SARS-

$280 \mathrm{CoV}-2$ variants of concern.

281

282 Methods

283 The sera of 101 healthcare workers with and without pre-existing immunity for

284 SARS-CoV-2 (as per former nasal swab positivity) who received their first vaccine

285 dose (BNT162b2 mRNA, Pfizer-BioNTech) in January 2021 were analyzed. Samples

286 had been collected and stored in the University of Verona biobank (Ethics Committee

287 approval prot. N. 1538) and in Tropica Biobank of the IRCCS Sacro Cuore Don

288 Calabria Hospital (Ethics Committee approval prot. N. 50950). All participants signed

289 informed consent.

290 The SARS-CoV-2 IgG-N assay and the SARS-CoV-2 IgM-S assay (Abbott, Ireland)

291 are chemiluminescent microparticle immunoassays (CMIA) used to detect IgG

292 antibodies to the nucleocapsid protein and $\operatorname{IgM}$ antibodies to the spike protein,

293 respectively, of SARS-CoV-2 in human serum. The automated assay was performed

294 according to the manufacturer's procedure, using the ARCHITECT I System

295 (Abbott). The resulting chemiluminescent reaction was measured as a relative light

296 unit (RLU) by the system optics. The RLU of the sample (S) was automatically

297 compared with the RLU of a specific calibrator I, resulting in an assay index (S/C). 
As per manufacturer's instructions, the interpretation of the results were as follow: index $(\mathrm{S} / \mathrm{C})<1.4=$ negative, index $(\mathrm{S} / \mathrm{C}) \geq 1.4$ = positive for $\mathrm{IgG}-\mathrm{N}$, and index $(\mathrm{S} / \mathrm{C})<1$

$300=$ negative, index $(\mathrm{S} / \mathrm{C}) \geq 1=$ positive, for IgM-S.

301 Serum samples were tested for the presence of SARS-CoV-2 IgA using the Anti-

302 SARS-CoV-2 ELISA kit (EUROIMMUN Medizinische Labordiagnostika AG,

303 Germany). The assay detects IgA antibodies in serum binding the S1 domain of the

304 SARS-CoV-2 spike protein. Samples were tested and analyzed as recommended by

305 the manufacturer, and results reported as a ratio based on sample O.D. divided by the 306 O.D. of the calibrators. Antibodies were considered undetectable (negative result) if

307 the ratio was less than 0.8 , borderline (inconclusive) between 0.8 and 1.1 , and positive 308 if greater 1.1.

309 The SARS-CoV-2 IgG II Quant assay (Abbott, Ireland) is a CMIA used for the 310 quantitative measure of IgG-S(RBD) antibodies (including neutralizing Abs) in 311 human serum. The automated assay was performed according to the manufacturer's 312 procedure, using the ARCHITECT I System (Abbott). Results were reported as 313 Arbitrary Unit (AU)/mL, according to the following interpretation: $\mathrm{AU} / \mathrm{mL}<50=$ 314 negative, $\mathrm{AU} / \mathrm{mL} \geq 50=$ positive. According to the WHO International Standard for 315 anti-SARS-CoV-2 immunoglobulin binding antibody units (BAU), the AU/mL are 316 converted into BAU by the equation: $\mathrm{AU} / \mathrm{mL}^{*} 0.142=\mathrm{BAU} / \mathrm{mL}$.

317 Lentiviral particles pseudotyped with SARS-CoV-2 spike were produced in $10 \mathrm{~cm}$ 318 plates seeded the day before with 3 million HEK293T cells in $10 \mathrm{ml}$ of complete

319 DMEM, supplemented with 10\% FBS. Cells were transfected using the Calcium 320 Phosphate technique with $15 \mu \mathrm{g}$ of an Env-defective SIV-Mac239 provirus construct 321 expressing GFP in place of $\mathrm{Nef}^{35}$ and $1.5 \mu \mathrm{g}$ PCDNA3.1 expression vector encoding 322 the WT SARS-CoV-2 spike (reference sequence Wuhan-Hu-1, accession number 
323 YP_009724390) with a truncation of the C-terminal 19 amino acids. Supernatants

324 containing pseudotyped virions were harvested 48 hours post-transfection, filtered

325 through a $0.45-\mu \mathrm{m}$ filter, and frozen at $-80^{\circ} \mathrm{C}$ until used. Sera neutralization titers

326 were assayed on Huh-7 cells engineered to overexpress the SARS-CoV-2 receptor

327 ACE2 upon stable transduction with a lentiviral expression vector. Target cells were

328 seeded on 384-well tissue culture plates one day before neutralization. Virus inoculum

329 was adjusted to produce no more than $10 \%$ of monolayer transduction to ensure a

330 linear working range of the assay. Sera dilutions were added to target cells using an

331 acoustic dispenser (Beckman Echo 650) to reach the indicated dilution in DMEM

332 with 10\% FBS. Pseudotyped virus was then added to wells using a Tecan Evo® 200

333 liquid handler. After 48 hours, transduction was assessed by calculating the

334 percentage of GFP-expressing cells upon nuclei counterstaining with Hoechst 33342

335 and measuring using the High Content Molecular Device Image Xpress ${ }^{\circledR}$ Micro

336 Confocal. Each serum dilution was evaluated in triplicate. Neutralization was

337 measured by calculating the residual transduction activity of the pseudovirus

338 considering the untreated sample as 100\%. Fitted sigmoidal curves and IC50 were

339 obtained using Prism (Graphpad) with the least square variable slope method and

340 using the normalized dose response protocol.

342 Statistical analysis

343 P values were calculated using the non-parametric two-tailed unpaired Kruskal-Wallis

344 test (Fig. 1 and Fig. 2), the two-sided Spearmen rank-correlation test (Fig. 3), the

345 Wilcoxon matched-pairs signed ranked test (Fig. 4), and the chi-squared test (Table 1)

346 using SPSS (version 22, SPSS Inc.) and Prism 9 (GraphPad Software, LLC). 


\section{Reporting Summary}

349 Further information on research design is available in the Nature Research Reporting

350 Summary linked to this article.

\section{Data availability}

353 Requests for data may be directed to the corresponding authors. Data limitations are

354 designed to ensure patients and participant confidentiality.

\section{Acknowledgments:}

357 This work was supported by FUR 2020 Department of Excellence 2018-2022, MIUR, 358 Italy, and the Brain Research Foundation Verona (D.Z.), the Italian Ministry of Health 359 under "Fondi Ricerca Corrente - L1P5" and "Progetto COVID Ricerca Finalizzata 360202012371675 ” to IRCCS Sacro Cuore Don Calabria Hospital (Z.B., C.P., N.T.,

361 S.L.), Fondazione VRT/CARITRO (M.P., S.A.), the COVID Research Projects 2020, 362 Italian Ministry of Health, COVID2020-12371617 (L.L.). We acknowledge the 363 generous contribution of our health care workers colleagues, whose sera samples were 364 essential to this study.

\section{Author Contributions:}

367 L.D.C. designed the study, enrolled patients, analyzed data and discussed results;

368 M.T.V. designed the study, collected samples and clinical data; Z.B. and C.P. 369 designed the study, collected data, managed data and critically revised the manuscript;

370 M.P. and S.A. performed and analyzed pseudovirus neutralization assays; S.M. , S.F., 371 D.A. L.M., N.T. and S.S.L. participated in data collection and analysis; A.M., J.B., 372 V.L.V., G.D. and E.C. collected samples and clinical data; L.L. analyzed and 
373 discussed data and critically revised the manuscript; A.B. analyzed and discussed

374 data, drafted and wrote the manuscript; D.Z. designed and coordinated the study,

375 analyzed data and results, prepared figures and tables, drafted and wrote the

376 manuscript.

377

378 Competing Interests Statement

379 All authors declare no competing financial interests.

$381 \quad$ References

382

383 1. Widge, A.T., et al. Durability of Responses after SARS-CoV-2 mRNA-1273

$384 \quad$ Vaccination. N Engl J Med 384, 80-82 (2021).

385 2. Ramasamy, M.N., et al. Safety and immunogenicity of ChAdOx1 nCoV-19

386 vaccine administered in a prime-boost regimen in young and old adults

387 (COV002): a single-blind, randomised, controlled, phase 2/3 trial. Lancet 396, 388 1979-1993 (2021).

389 3. Anichini, G., et al. SARS-CoV-2 Antibody Response in Persons with Past

$390 \quad$ Natural Infection. N Engl J Med Epub ahead of print March 1 (2021).

391 4. Harvey, R.A., et al. Association of SARS-CoV-2 Seropositive Antibody Test

392 With Risk of Future Infection. JAMA Intern Med Epub ahead of print

$393 \quad$ February 24 (2021).

394 5. Lumley, S.F., et al. Antibody Status and Incidence of SARS-CoV-2 Infection 395 in Health Care Workers. N Engl J Med 384, 533-540 (2021). 
396 6. Melgaco, J.G., Azamor, T. \& Ano Bom, A.P.D. Protective immunity after COVID-19 has been questioned: What can we do without SARS-CoV-2-IgG detection? Cell Immunol 353, 104114 (2020).

7. Ebinger, J.E., et al. Antibody responses to the BNT162b2 mRNA vaccine in 400 individuals previously infected with SARS-CoV-2. Nat Med Epub ahead of $401 \quad$ print April 1 (2021).

402 8. Krammer, F., et al. Antibody Responses in Seropositive Persons after a Single Dose of SARS-CoV-2 mRNA Vaccine. N Engl J Med 384, 1372-1374 (2021).

$4049 . \quad$ Bradley, T., et al. Antibody Responses after a Single Dose of SARS-CoV-2 mRNA Vaccine. $N$ Engl J Med Epub ahead of print February 5 (2021).

406 10. Saadat, S., et al. Binding and Neutralization Antibody Titers After a Single Vaccine Dose in Health Care Workers Previously Infected With SARS-CoV2. JAMA 325, 1467-1469 (2021).

409 11. Beretta, A., Cranage, M. \& Zipeto, D. Is Cross-Reactive Immunity Triggering $410 \quad$ COVID-19 Immunopathogenesis? Front Immunol 11, 567710 (2020).

411 12. Levi, R., et al. A cautionary note on recall vaccination in ex-COVID-19 subjects. medRxiv, 2021.2002.2001.21250923 (2021).

413 13. Stamatatos, L., et al. mRNA vaccination boosts cross-variant neutralizing 414 antibodies elicited by SARS-CoV-2 infection. Science, eabg9175 (2021).

415 14. Ng, K.W., et al. Preexisting and de novo humoral immunity to SARS-CoV-2 416 in humans. Science 370, 1339-1343 (2020).

417 15. Nguyen-Contant, P., et al. S Protein-Reactive IgG and Memory B Cell 418 Production after Human SARS-CoV-2 Infection Includes Broad Reactivity to 419 the S2 Subunit. mBio 11, 2020.2007.2020.213298 (2020). 
420 16. Atyeo, C., et al. Distinct Early Serological Signatures Track with SARS-CoV4212 Survival. Immunity 53, 524-532 e524 (2020).

422 17. Gorse, G.J., Donovan, M.M. \& Patel, G.B. Antibodies to coronaviruses are higher in older compared with younger adults and binding antibodies are more sensitive than neutralizing antibodies in identifying coronavirus-associated illnesses. J Med Virol 92, 512-517 (2020).

426 18. Lv, H., et al. Cross-reactive Antibody Response between SARS-CoV-2 and SARS-CoV Infections. Cell Reports 31, 107725 (2020).

19. Nielsen, S.C.A., et al. Human B Cell Clonal Expansion and Convergent Antibody Responses to SARS-CoV-2. Cell Host Microbe 28, 516-525 e515 (2020).

20. Seow, J., et al. Longitudinal observation and decline of neutralizing antibody responses in the three months following SARS-CoV-2 infection in humans. Nat Microbiol 5, 1598-1607 (2020).

434 21. Long, Q.X., et al. Antibody responses to SARS-CoV-2 in patients with COVID-19. Nat Med 26, 845-848 (2020).

22. Pisanic, N., et al. COVID-19 Serology at Population Scale: SARS-CoV-2Specific Antibody Responses in Saliva. Journal of Clinical Microbiology 59, e02204-02220 (2020).

439 23. Sun, B., et al. Kinetics of SARS-CoV-2 specific IgM and IgG responses in 440 COVID-19 patients. Emerg Microbes Infect 9, 940-948 (2020).

441 24. Cervia, C., et al. Systemic and mucosal antibody responses specific to SARS442 CoV-2 during mild versus severe COVID-19. Journal of Allergy and Clinical Immunology 147, 545-557.e549 (2021). 
25. Ma, H., et al. Serum IgA, IgM, and IgG responses in COVID-19. Cell Mol Immunol 17, 773-775 (2020).

446 26. Yu, H.Q., et al. Distinct features of SARS-CoV-2-specific IgA response in COVID-19 patients. Eur Respir J 56(2020).

448 27. Gobbi, F., et al. Antibody Response to the BNT162b2 mRNA COVID-19 Vaccine in Subjects with Prior SARS-CoV-2 Infection. Viruses 13, 422 (2021).

451 28. Mazzini, L., et al. Comparative analyses of SARS-CoV-2 binding (IgG, IgM, IgA) and neutralizing antibodies from human serum samples. J Immunol Methods 489, 112937 (2021).

454 29. Cervia, C., et al. Systemic and mucosal antibody secretion specific to SARSCoV-2 during mild versus severe COVID-19. bioRxiv, 2020.2005.2021.108308 (2020).

30. Gluck, V., et al. SARS-CoV-2-directed antibodies persist for more than six months in a cohort with mild to moderate COVID-19. Infection Epub ahead of print March 10 (2021).

460 31. Gaebler, C., et al. Evolution of antibody immunity to SARS-CoV-2. Nature 591, 639-644 (2021).

462 32. Wec, A.Z., et al. Broad neutralization of SARS-related viruses by human 463 monoclonal antibodies. Science 369, 731-736 (2020).

464 33. Wang, Z., et al. Enhanced SARS-CoV-2 neutralization by dimeric IgA. Sci 465 Transl Med 13, eabf1555 (2021).

466 34. Zohar, T., et al. Compromised Humoral Functional Evolution Tracks with SARS-CoV-2 Mortality. Cell 183, 1508-1519 e1512 (2020). 
468 35. Pizzato, M., et al. Lv4 Is a Capsid-Specific Antiviral Activity in Human Blood 469 Cells That Restricts Viruses of the SIVMAC/SIVSM/HIV-2 Lineage Prior to

470 Integration. PLoS Pathog 11, e1005050 (2015).

471 
Table 1. Characteristics of the study population

475

\begin{tabular}{|l|c|c|c|}
\hline & Naïve (50) & P.I. (51) & p \\
\hline Males/Females & $13 / 37$ & $19 / 32$ & $\mathrm{p}=0.23$ (ns) \\
\hline Age (yrs) & $43 \pm 13$ & $46 \pm 12$ & $\mathrm{p}=0.21$ (ns) \\
\hline Fever after 1 ${ }^{\text {st }}$ dose (\%) & 7.7 & 0 & $\mathrm{p}=0.40$ (ns) \\
\hline Discomfort after $1^{\text {st }}$ dose (\%) & 26.9 & 22.2 & $\mathrm{p}=0.78(\mathrm{~ns})$ \\
\hline Fever after $2^{\text {nd }}$ dose (\%) & 38.5 & 33.3 & $\mathrm{p}=0.79$ (ns) \\
\hline Discomfort after $2^{\text {nd }}$ dose (\%) & 53.8 & 66.7 & $\mathrm{p}=0.51(\mathrm{~ns})$ \\
\hline
\end{tabular}

476

477 P.I: Previously infected; ns: not significant

478 
Table 2. Antibody and neutralization assays (positives/total and percent)

\begin{tabular}{|l|l|l|l|l|l|}
\hline Test & Time & Naïve & P.I. & $\mathbf{1}^{\text {st }}$ wave & $2^{\text {nd }}$ wave 480 \\
\hline \multirow{2}{*}{ IgG-N } & T0 & $0 / 48(0 \%)$ & $24 / 51(47.1 \%)$ & $7 / 25(28 \%)$ & $17 / 26(65.4 \%)$ \\
& T1 & $0 / 50(0 \%)$ & $23 / 51(45.1 \%)$ & $7 / 25(28 \%)$ & $16 / 26(61.5 \%)$ \\
& T2 & $0 / 49(0 \%)$ & $21 / 49(42.9 \%)$ & $6 / 25(24 \%)$ & $14 / 24(58.3 \%)$ \\
\hline \multirow{2}{*}{ IgM-S } & T0 & $0 / 48(0 \%)$ & $24 / 51(47.1 \%)$ & $8 / 25(32 \%)$ & $16 / 26(61.54 \%)$ \\
& T1 & $27 / 50(54 \%)$ & $23 / 51(45.1 \%)$ & $8 / 25(32 \%)$ & $15 / 26(57.7 \% \%)$ \\
\hline \multirow{2}{*}{ IgG-S(RBD) } & T2 & $29 / 49(59.2 \%)$ & $15 / 49(30.6 \%)$ & $5 / 25(20 \%)$ & $10 / 24(41.7 \%)$ \\
& T0 & $0 / 48(0 \%)$ & $46 / 51(90.2 \%)$ & $23 / 25(92 \%)$ & $23 / 26(88.5 \%)$ \\
\hline \multirow{2}{*}{ IgA-S } & T2 & $49 / 50(98 \%)$ & $50 / 51(98 \%)$ & $25 / 25(100 \%)$ & $25 / 26(96.2 \%)$ \\
& T0 & $0 / 49(0 \%)$ & $40 / 51(78.4 \%)$ & $18 / 25(72 \%)$ & $22 / 26(84.6 \%)$ \\
& T1 & $44 / 50(88 \%)$ & $51 / 51(100 \%)$ & $25 / 25(100 \%)$ & $26 / 26(100 \%)$ \\
& T2 & $49 / 49(100 \%)$ & $49 / 49(100 \%)$ & $25 / 25(100 \%)$ & $24 / 24(100 \%)$ \\
\hline \multirow{2}{*}{ TCID50 } & T0 & $2 / 48(4.2 \%)$ & $46 / 51(90.2 \%)$ & $23 / 25(92 \%)$ & $23 / 26(88.5 \%)$ \\
& T1 & $47 / 50(94 \%)$ & $51 / 51(100 \%)$ & $25 / 25(100 \%)$ & $26 / 26(100 \%)$ \\
& T2 & $49 / 49(100 \%)$ & $50 / 50(100 \%)$ & $25 / 25(100 \%)$ & $25 / 25(100 \%)$ \\
\hline
\end{tabular}


Table 3. Number and percent of IgM-S and IgG-S(RBD) positive subjects

496

\begin{tabular}{|c|c|c|c|c|c|c|c|c|c|c|c|c|c|}
\hline \multirow[b]{2}{*}{ IgM } & \multirow[b]{2}{*}{ IgG } & \multicolumn{3}{|l|}{ Naïve } & \multicolumn{3}{|l|}{ P.I. } & \multicolumn{3}{|l|}{$1^{\text {st }}$ wave } & \multicolumn{3}{|l|}{$2^{\text {nd }}$ wave } \\
\hline & & T0 & T1 & T2 & T0 & T1 & $\mathbf{T} 2$ & T0 & T1 & T2 & T0 & T1 & $\mathbf{T} 2$ \\
\hline+ & + & $0(0 \%)$ & $27(54 \%)$ & $29(59.2 \%)$ & $24(47.1 \%)$ & $22(43.1 \%)$ & $15(30.6 \%)$ & $8(32 \%)$ & $8(32 \%)$ & $5(20 \%)$ & $16(61.5 \%)$ & $14(53.8 \%)$ & $10(41.7 \%)$ \\
\hline+ & - & $0(0 \%)$ & $0(0 \%)$ & $0(0 \%)$ & $0(0 \%)$ & $1(2 \%)$ & $0(0 \%)$ & $0(0 \%)$ & $0(0 \%)$ & $0(0 \%)$ & $0(0 \%)$ & $1(3.8 \%)$ & $0(0 \%)$ \\
\hline - & + & $1(2.1 \%)$ & $23(46 \%)$ & $20(40.8 \%)$ & $22(43.1 \%)$ & $28(54.9 \%)$ & $34(69.4 \%)$ & $15(60 \%)$ & $17(68 \%)$ & $20(80 \%)$ & $7(26.9 \%)$ & $1142.3 \%)$ & $14(58.3 \%)$ \\
\hline- & - & $47(97.9 \%)$ & $0(0 \%)$ & $0(0 \%)$ & $5(9.8 \%)$ & $0(0 \%)$ & $0(0 \%)$ & $2(8 \%)$ & $0(0 \%)$ & $0(0 \%)$ & $3(11.5 \%)$ & $0(0 \%)$ & $0(0 \%)$ \\
\hline
\end{tabular}

497

498 P.I.: Previously Infected

499 
Fig. 1: analysis of the antibody response profile at the time of first vaccination (T0), second vaccination (T1), and 3 weeks after the boost (T2) in naïve and previously infected (P.I.) recipients. Median values with the interquartile range are displayed. The horizontal dot lines indicate the limit of each assay, according to the manufacturer's instructions. Panel A: IgG for the SARS-CoV-2 nucleocapsid protein $\mathrm{N}$; panel B: IgM for the spike glycoprotein; panel C: IgA for the spike glycoprotein; panel D: IgG for the receptor-binding domain (RBD) of the spike; panel E:

509 pseudoviruses neutralization assay, expressed as median tissue culture infectious dose

510 (TCID50). Sample sizes are reported in Table 2. P values were calculated using the 511 non-parametric, two-tailed unpaired Kruskal-Wallis test. Differences were considered 512 significant if $\mathrm{p}<0.05$.

514 Figure 2: analysis of the antibody response profile at the time of first vaccination 515 (T0), second vaccination (T1) and 3 weeks after (T2) in subjects infected during the first (orange dots) and the second (red dots) COVID-19 wave. Median values

517 with the interquartile range are displayed; the horizontal dot lines indicate the limit of 518 each assay, according to the manufacturer's instructions. Panel A: IgG for the SARS-

519 CoV-2 nucleocapsid protein N; panel B: IgM for the spike glycoprotein; panel C: IgA 520 for the spike glycoprotein; panel D: IgG for the receptor-binding domain (RBD) of 521 the spike; panel E: neutralization assay, expressed as median tissue culture infectious 522 dose (TCID50). Sample sizes are reported in Table 2. P values were calculated using 523 the non-parametric, two-tailed unpaired Kruskal-Wallis test. Differences were 524 considered significant if $\mathrm{p}<0.05$. 
526 Figure 3: analysis of the correlation at time of first vaccination (T0), second

527 vaccination (T1), and 3 weeks after (T2) in naïve (top row) and previously

528 infected (P.I.) recipients (bottom row). Panel A: correlation between IgG-S(RBD)

529 and neutralization (TCID50); panel B: correlation between IgG-S(RBD) and IgA;

530 panel C: correlation between neutralization (TCID50) and IgA-S. Sample sizes are

531 reported in Table 2. The correlation was calculated using the two-sided Spearmen

532 rank-correlation test.

533

534 Figure 4: antibody response in naïve and previously infected (P.I.) male (blue

535 dots) and female (red dots) subjects following vaccination. Panel A: IgG-S(RBD)

536 titers at the time of first vaccination (T0, n. males/females 12/36 for naïve, 19/32 for

537 P.I.), second vaccination (T1, n. males/females 13/37 for naïve, 19/32 for P.I), and 3

538 weeks after the boost (T2, n. males/females 13/36 for naïve, 19/30 for P.I); panel B:

539 neutralization activity expressed as TCID50 (n. males/females: T0 12/36, T1 13/37,

540 T2 13/36 for naïve, T0 19/32, T1 19/32, T2 19/31 for P.I.); panel C: IgA-S antibody

541 titers (n. males/females: T0 12/37, T1 13/37, T2 13/36 for naïve, T0 19/32, T1 19/32,

542 T2 18/31 for P.I.). P-values were calculated using the Wilcoxon matched-pairs signed

543 ranked test and considered significant if $\mathrm{p}<0.05$. 
Figures

$$
A-\operatorname{lgG}-\mathrm{N}
$$

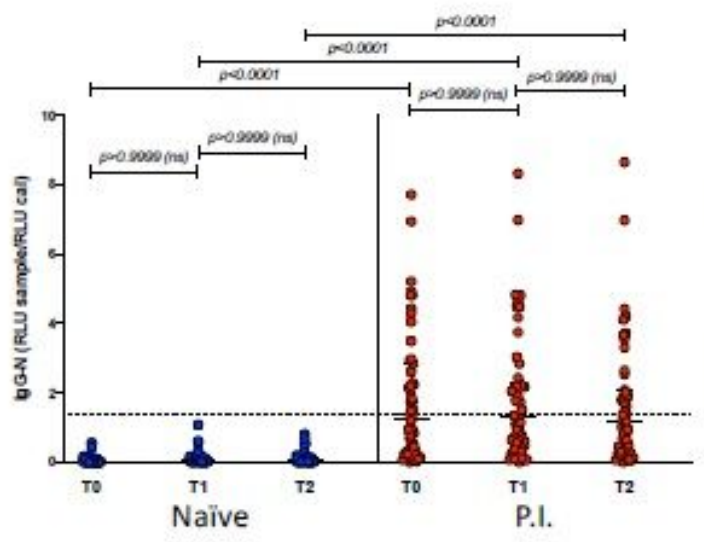

C - IgA-S

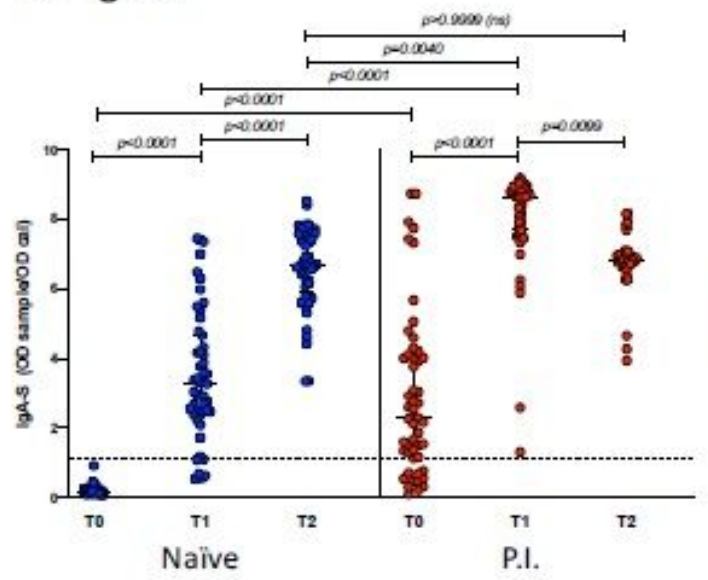

\section{E - TCID50}

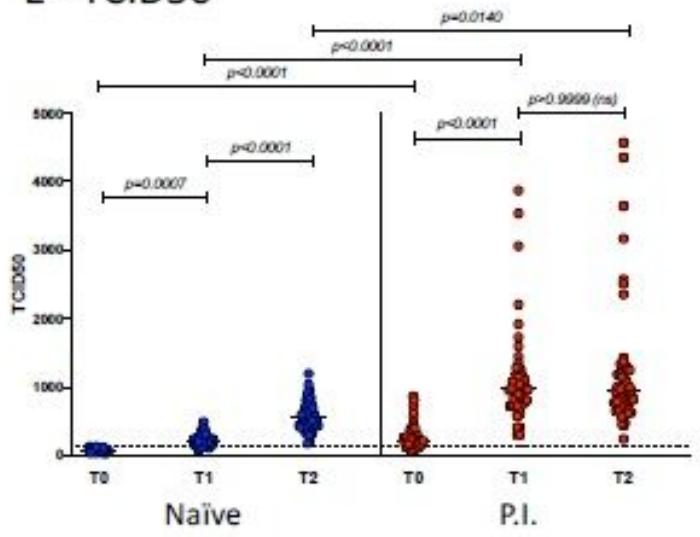

B - IgM-S
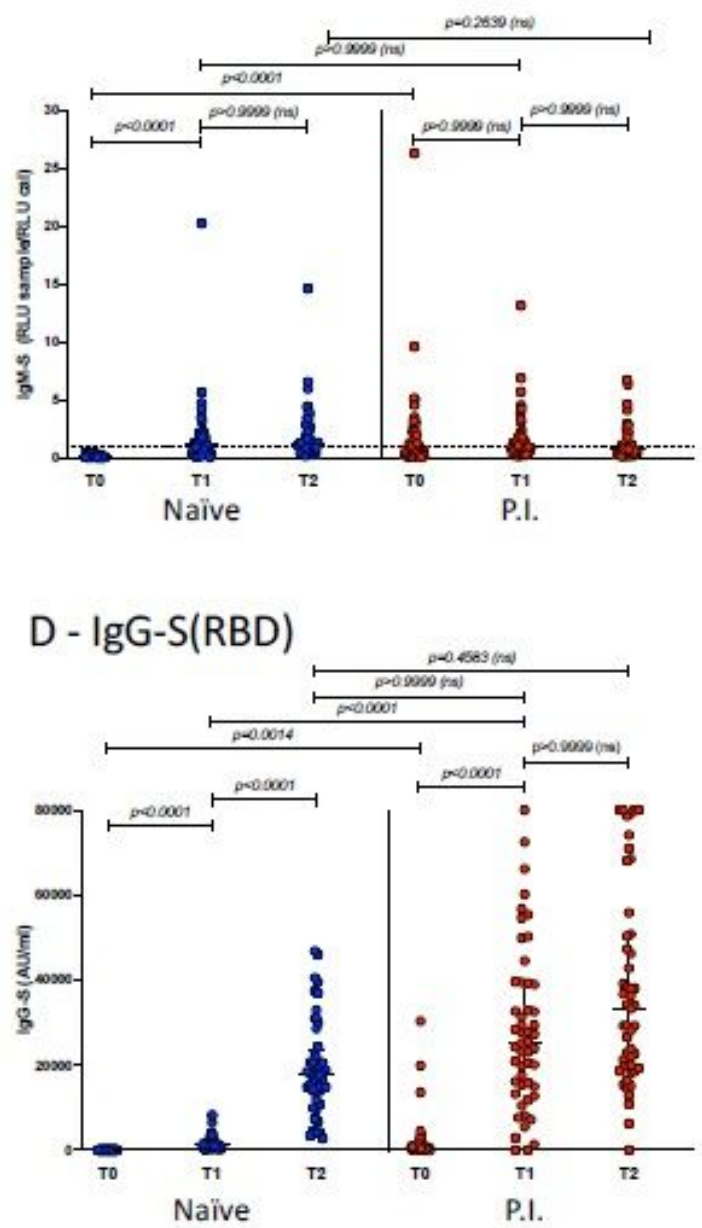

\section{Figure 1}

analysis of the antibody response profile at the time of first vaccination (T0), second vaccination (T1), and 3 weeks after the boost (T2) in naïve and previously infected (P.I.) recipients. Median values with the interquartile range are displayed. The horizontal dot lines indicate the limit of each assay, according to 
the manufacturer's instructions. Panel A: IgG for the SARS-CoV-2 nucleocapsid protein N; panel B: IgM for the spike glycoprotein; panel C: IgA for the spike glycoprotein; panel D: IgG for the receptor-binding domain (RBD) of the spike; panel E: pseudoviruses neutralization assay, expressed as median tissue culture infectious dose (TCID50). Sample sizes are reported in Table 2. P values were calculated using the non-parametric, two-tailed unpaired Kruskal-Wallis test. Differences were considered significant if $p<0.05$.
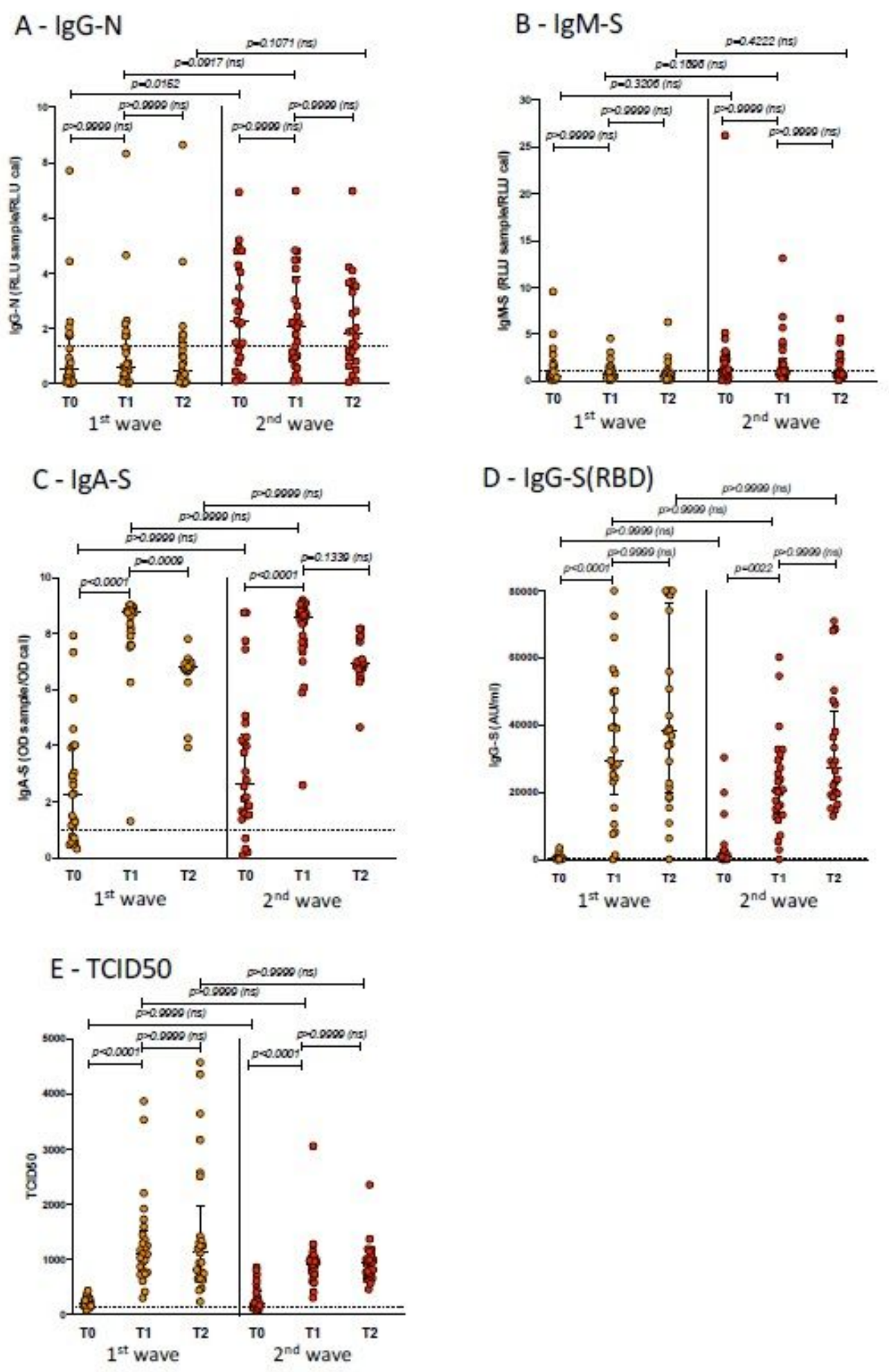

Figure 2 
analysis of the antibody response profile at the time of first vaccination (T0), second vaccination (T1) and 3 weeks after (T2) in subjects infected during the first (orange dots) and the second (red dots) COVID-19 wave. Median values with the interquartile range are displayed; the horizontal dot lines indicate the limit of each assay, according to the manufacturer's instructions. Panel A: IgG for the SARS-CoV-2 nucleocapsid protein N; panel B: IgM for the spike glycoprotein; panel C: IgA for the spike glycoprotein; panel $\mathrm{D}$ : IgG for the receptor-binding domain (RBD) of the spike; panel E: neutralization assay, expressed as median tissue culture infectious dose (TCID50). Sample sizes are reported in Table 2. P values were calculated using the non-parametric, two-tailed unpaired Kruskal-Wallis test. Differences were considered significant if $p<0.05$. 
A - TCID50 vs IgG-S(RBD)
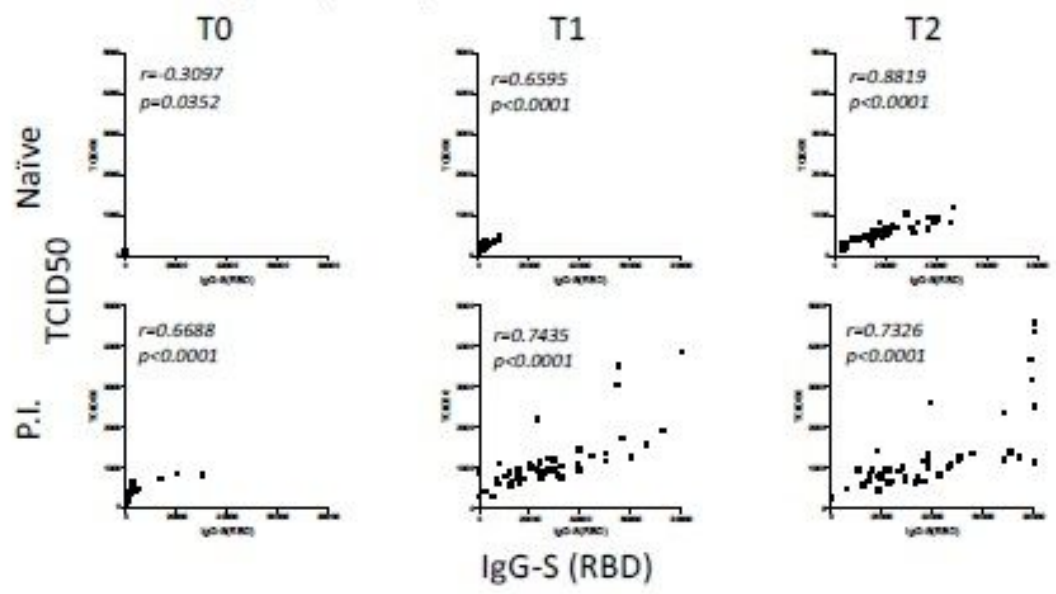

B - IgA-S vs IgG-S(RBD)
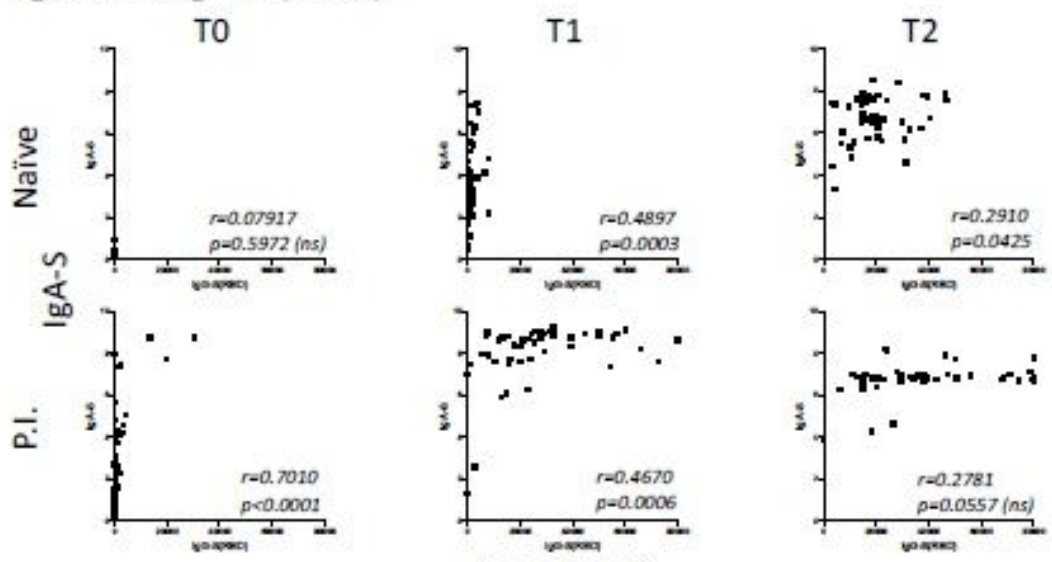

\section{C - IgA-S vs TCID50}
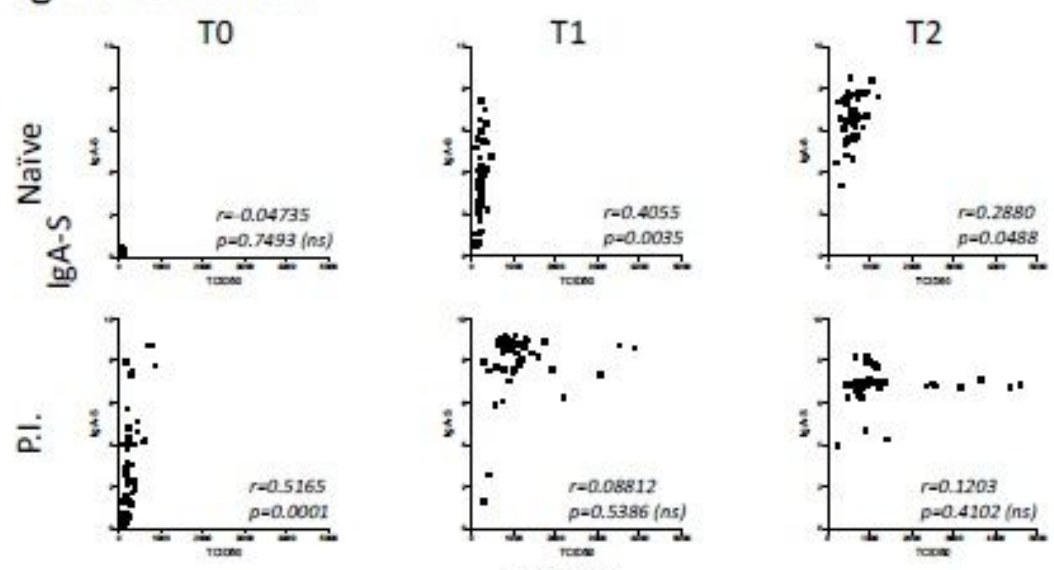

TCID50

\section{Figure 3}

analysis of the correlation at time of first vaccination (T0), second vaccination (T1), and 3 weeks after (T2) in naïve (top row) and previously infected (P.I.) recipients (bottom row). Panel A: correlation between IgG-S(RBD) and neutralization (TCID50); panel B: correlation between IgG-S(RBD) and IgA; panel C: correlation between neutralization (TCID50) and IgA-S. Sample sizes are reported in Table 2. The correlation was calculated using the two-sided Spearmen rank-correlation test. 
A - IgG-S(RBD)

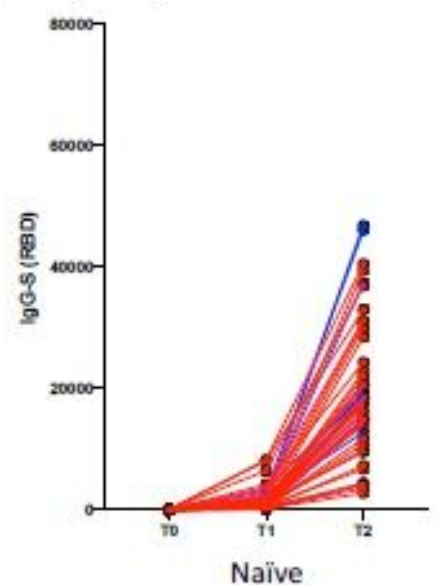

B - TCID50

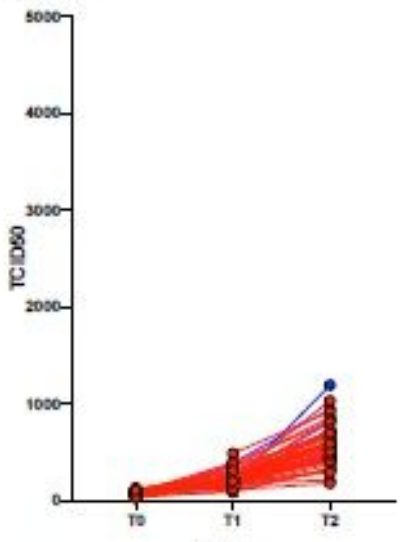

Naïve

\section{C - IgA-S}

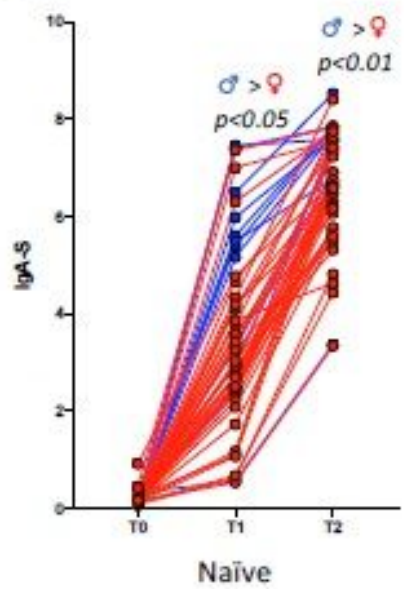

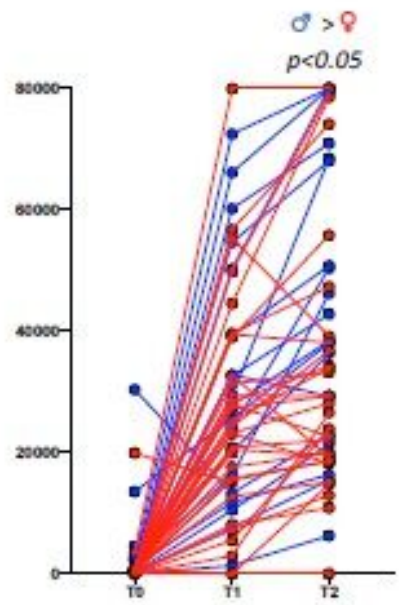

P.I.

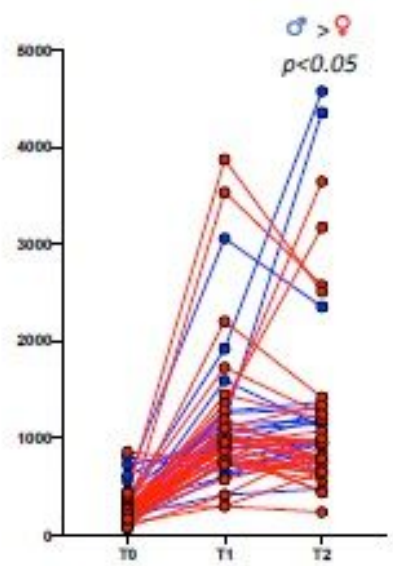

P.I.

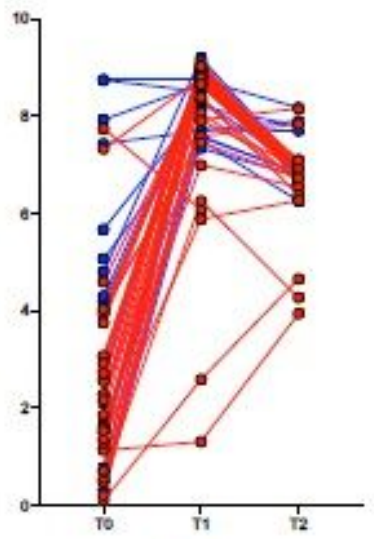

P.I.

\section{Figure 4}

antibody response in naïve and previously infected (P.I.) male (blue dots) and female (red dots) subjects following vaccination. Panel A: IgG-S(RBD) titers at the time of first vaccination (T0, n. males/females 12/36 for naïve, 19/32 for P.I.), second vaccination (T1, n. males/females 13/37 for naïve, 19/32 for P.I), and 3 weeks after the boost (T2, n. males/females $13 / 36$ for naïve, 19/30 for P.I); panel B: neutralization activity expressed as TCID50 (n. males/females: T0 12/36, T1 13/37, T2 13/36 for naïve, T0 19/32, T1 
19/32, T2 19/31 for P.I.); panel C: IgA-S antibody titers (n. males/females: T0 12/37, T1 13/37, T2 13/36 for naïve, T0 19/32, T1 19/32, T2 18/31 for P.I.). P-values were calculated using the Wilcoxon matchedpairs signed ranked test and considered significant if $p<0.05$. 